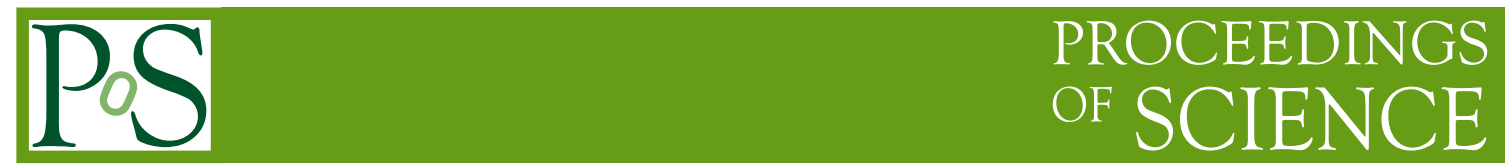

\title{
Search for D and B leptonic decays at Belle
}

\section{Marko Starič ${ }^{* \dagger}$}

J. Stefan Institute, Ljubljana, Slovenia

E-mail: marko.stariceijs.si

Based on $660 \mathrm{fb}^{-1}$ of data collected in $e^{+} e^{-}$collisions at the centre-of-mass energy of the $\Upsilon(4 S)$ resonance and $60 \mathrm{MeV}$ below by the Belle detector at the KEKB collider, we search for flavourchanging neutral current decays $D^{0} \rightarrow \mu^{+} \mu^{-}$and $D^{0} \rightarrow e^{+} e^{-}$, and for lepton-flavour violating decays $D^{0} \rightarrow e^{ \pm} \mu^{\mp}$.

35th International Conference of High Energy Physics

July 22-28, 2010

Paris, France

* Speaker.

${ }^{\dagger}$ on behalf of Belle collaboration 


\section{Introduction}

In the standard model (SM) the flavour-changing neutral current (FCNC) decays $D^{0} \rightarrow e^{+} e^{-}$ and $D^{0} \rightarrow \mu^{+} \mu^{-}$[1] are highly suppressed by the Glashow-Iliopoulos-Maiani mechanism [2]. Branching fractions for such modes in the SM are around $10^{-13}$, considering long distance contributions [3]. The SM forbids lepton-flavour violating (LFV) decays $D^{0} \rightarrow e^{ \pm} \mu^{\mp}$, however they are possible in extensions of the SM with massive neutrinos and the branching fractions in such cases are expected to be of the order of $10^{-14}$ [3]. At the current experimental sensitivity we are unable to observe any of these decays.

Contributions from new physics (NP) scenarios can enhance FCNC branching fractions by many orders of magnitude. For example, $R$-parity violating supersymmetry can enhance the branching fractions of $D^{0} \rightarrow e^{+} e^{-}$and $D^{0} \rightarrow \mu^{+} \mu^{-}$up to $10^{-12}$ and $10^{-8}$, respectively [4]. The current experimental sensitivity is close to the latter prediction. As a way to explain the small discrepancy between the measured value of the $D_{s}$ meson decay constant and the prediction of lattice QCD so far unobserved leptoquarks were suggested [5]. To comply with constraints from charm physics and ascribe the decay constant discrepancy to a leptoquark contribution, $\mathscr{B}\left(D^{0} \rightarrow \mu^{+} \mu^{-}\right)$should be enhanced up to $8 \times 10^{-7}$ [6].

\section{Measurement}

Based on a data sample of $660 \mathrm{fb}^{-1}$ of data recorded by the Belle detector at the KEKB collider, we searched for the decays $D^{0} \rightarrow \mu^{+} \mu^{-}, D^{0} \rightarrow e^{+} e^{-}$and $D^{0} \rightarrow e^{ \pm} \mu^{\mp}$. To reduce combinatorial background we consider in the analysis only $D^{0}$ mesons coming from $c$ - quark production in the continuum $e^{+} e^{-} \rightarrow c \bar{c}$. Only $D^{0}$ mesons coming form $D^{*+} \rightarrow D^{0} \pi^{+}$decays are considered, since this considerably improves the purity of the reconstructed samples. To cancel various systematic uncertainties we normalise the sensitivity of our search to topologically similar $D^{0} \rightarrow \pi^{+} \pi^{-}$decays. The signal efficiencies $\varepsilon_{\ell \ell}$ and $\varepsilon_{\pi \pi}$ are evaluated using signal Monte Carlo simulation, based on EVTGEN [8] and GEANT3 [9].

In order to avoid biases, a blind analysis technique has been adopted. The decays we are searching for $D^{0} \rightarrow \ell^{+} \ell^{-}$decays are not expected to be observed at the current sensitivity, consequently we maximise the figure-of-merit, $\mathscr{F}=\varepsilon_{\ell \ell} / N_{\mathrm{UL}}$, where $\varepsilon_{\ell \ell}$ denotes the efficiency for reconstructing $D^{0} \rightarrow \ell^{+} \ell^{-}$decays, and $N_{\mathrm{UL}}$ is the Poisson average of Feldman-Cousins $90 \%$ confidence level upper limits on the number of observed signal events that would be obtained with the expected background and no signal [12].

We categorize the background into two groups: (1) a smooth combinatorial background, and (2) a peaking background from the misidentification of $D^{0} \rightarrow \pi^{+} \pi^{-}$decays. The sideband region is used to determine the number of combinatorial background events in the signal region.

The signal like background due to misidentification of $D^{0} \rightarrow \pi^{+} \pi^{-}$decays is estimated in two steps. Firstly, we reconstruct $D^{0} \rightarrow \pi^{+} \pi^{-}$decays in data so that the pion mass is replaced with the lepton mass. Secondly, each event is weighted with the pion-lepton misidentification probability and pion identification efficiency. The misidentification probabilities and efficiencies are measured in data using $D^{*+} \rightarrow D^{0} \pi^{+}, D^{0} \rightarrow K^{-} \pi^{+}$decays, binned in particle momentum $p$ and cosine of 
polar angle. We find only a significant contribution of the misidentification of $D^{0} \rightarrow \pi^{+} \pi^{-}$decays in the $D^{0} \rightarrow \mu^{+} \mu^{-}$decay channel (1.8 events).

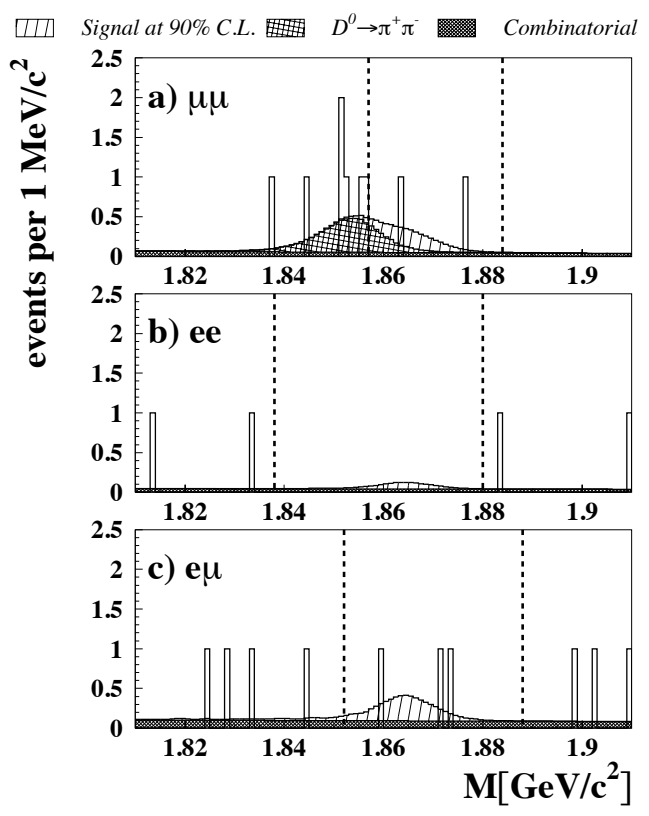

Figure 1: The invariant mass distributions for (a) $D^{0} \rightarrow \mu^{+} \mu^{-}$, (b) $D^{0} \rightarrow e^{+} e^{-}$, and (c) $D^{0} \rightarrow e^{ \pm} \mu^{\mp}$. Superimposed on the data (open histograms) are the estimated distribution for combinatorial background (filled histogram), the misidentification of $D^{0} \rightarrow \pi^{+} \pi^{-}$(cross-hatched histogram), and the signal if the branching fractions were equal to the $90 \%$ confidence level upper limit (single hatched histogram). The dashed vertical lines indicate the optimised signal window [10].

Figure 1 shows the invariant mass distributions after applying the optimised event selection criteria. We find in the signal region two candidates in the $D^{0} \rightarrow \mu^{+} \mu^{-}$, zero candidates in the $D^{0} \rightarrow e^{+} e^{-}$and three candidates in the $D^{0} \rightarrow e^{ \pm} \mu^{\mp}$ decay mode; the estimated backgrounds (3.1 $\pm 0.1,1.7 \pm 0.2$, and $2.6 \pm 0.2$ events respectively) are consistent with the yields in the signal window. For the means of normalisation a binned maximum likelihood fit is used to determine the number of $D^{0} \rightarrow \pi^{+} \pi^{-}$candidates. We use the programme pole.f [11], which extends the Feldman-Cousins method [12] by the inclusion of systematic uncertainties, to calculate the branching fraction upper limits.

\section{Conclusion}

Using the Belle detector we have searched for the FCNC decays $D^{0} \rightarrow \mu^{+} \mu^{-}$and $D^{0} \rightarrow e^{+} e^{-}$, and the LFV decays $D^{0} \rightarrow e^{ \pm} \mu^{\mp}$ and have found no evidence of these decays. The upper limits on the branching fractions at the $90 \%$ confidence level are $\mathscr{B}\left(D^{0} \rightarrow \mu^{+} \mu^{-}\right)<1.4 \times 10^{-7}$, $\mathscr{B}\left(D^{0} \rightarrow e^{+} e^{-}\right)<7.9 \times 10^{-8}$ and $\mathscr{B}\left(D^{0} \rightarrow e^{ \pm} \mu^{\mp}\right)<2.6 \times 10^{-7}$ [10]. In comparison to previously published results [7] our results represent an improvement by factor of 9 for $D^{0} \rightarrow \mu^{+} \mu^{-}$ decay, by a factor of 15 for $D^{0} \rightarrow e^{+} e^{-}$decay and by a factor of 3 for $D^{0} \rightarrow e^{ \pm} \mu^{\mp}$ decay. The 
CDF collaboration has recently published a result on the upper limit for the $D^{0} \rightarrow \mu^{+} \mu^{-}$branching fraction [13]; however our result still remain lower and can further constrain the parameters of NP scenarios. The result brings some tension into the explanation of the anomaly in the measured $D_{s}^{+} \rightarrow \mu^{+} v$ width with a leptoquark contribution [6].

\section{References}

[1] Throughout this paper charge-conjugate decay modes are included.

[2] S. L. Glashow, J. Iliopoulos and L. Maiani, Phys. Rev. D 2 (1970) 1285.

[3] G. Burdman, E. Golowich, J. L. Hewett and S. Pakvasa, Phys. Rev. D 66 (2002) 014009.

[4] E. Golowich, J. Hewett, S. Pakvasa and A. A. Petrov, Phys. Rev. D 79 (2009) 114030.

[5] B. A. Dobrescu and A. S. Kronfeld, Phys. Rev. Lett. 100 (2008) 241802.

[6] I. Doršner, S. Fajfer, J. F. Kamenik and N. Košnik, Phys. Lett. B 682 (2009) 67.

[7] C. Amsler et al. (Particle Data Group), Phys. Lett. B 667 (2008) 1.

[8] D. J. Lange, Nucl. Instrum. Meth. A 462, (2001) 152.

[9] R. Brun, F. Bruyant, M. Maire, A. C. McPherson, and P. Zanarini, (1987), CERN-DD/EE/84-1.

[10] M. Petrič et al. (Belle Collaboration), Phys. Rev. D 81 (2010) 091102.

[11] J. Conrad, O. Botner, A. Hallgren and C. Perez de los Heros, Phys. Rev. D 67 (2003) 012002.

[12] G. J. Feldman and R. D. Cousins, Phys. Rev. D 57 (1998) 3873.

[13] T. Aaltonen et al. (CDF Collaboration), Phys. Rev. D 82 (2010) 091105. 